\title{
How to Engineer Biologically Inspired Cognitive Architectures
}

\author{
Valeria Seidita $^{1}$, Massimo Cossentino $^{2}$, and Antonio Chella ${ }^{1}$ \\ 1 Dipartimento di Ingegneria Chimica, Gestionale, Informatica, Meccanica \\ University of Palermo, Palermo, Italy \\ \{valeria.seidita, antonio.chella\}@unipa.it \\ ${ }^{2}$ Istituto di Reti e Calcolo ad Alte Prestazioni, Consiglio Nazionale delle Ricerche \\ ICAR/CNR Palermo, Italy \\ cossentino@pa.icar.cnr.it
}

\begin{abstract}
Biologically inspired cognitive architectures are complex systems where different modules of cognition interact in order to reach the global goals of the system in a changing environment. Engineering and modeling this kind of systems is a hard task due to the lack of techniques for developing and implementing features like learning, knowledge, experience, memory, adaptivity in an inter-modular fashion. We propose a new concept of intelligent agent as abstraction for developing biologically cognitive architectures.
\end{abstract}

Researchers in different fields today are attracted by the idea of creating a society where human beings and artificial intelligent agents cohabit in a purposeful manner by sharing tasks and objectives. This is one of the main challenges of Biologically Inspired Cognitive Architecture (BICA) research community.

The full development of an intelligent organism goes through experience and reasoning (more or less consciously). An intelligent human being, capable of implementing cognitive behavior, is an entity who has gone through a series of moments where he learned from the experience and from interactions with the surrounding world. We claim that the surrounding world, or the environment, an intelligent organism lives in, is the set of human and non-human entities, artificial and not, conscious and not, which lives, exists and acts around it, and even within itself. Human-like intelligence is the result of a developmental process that occurs over time.

We highlight the fact that, so as an intelligent organism, a biologically cognitive system can not be developed from scratch and endowed with a "complete" intelligence, hence we have to design and develop cognitive systems with a minimum basic intelligence; eventually it must be made able to evolve according to the environment in which it lives.

In order to face this problem from an engineering point of view and in order to find means for creating and using software engineering techniques for developing such systems, we started from analyzing several existing cognitive architectures and from abstracting from them a set of common features. Among the most known we analyzed [1][3][4][7][8] and [5] for a general overview. 
We experienced that classical software engineering and also agent software engineering are not enough and do not posses the right abstractions for managing such kind of systems. Engineering and designing biologically cognitive architecture seen as cross-interacting modules, imply to identify the right abstractions a cognitive system presents.

The model of the cognitive system we propose considers that a human being, a simple organism, a society of individuals and in the same way their artificial counterpart (agent, from now on) have, in the whole life or in a part of it, one main goal that can be, if necessary, decomposed in sub-goals. Agent pursues its goal(s) by interacting with its environment both external, hence made of other agents, objects and so on, and internal, hence made of emotions, reasoning, knowledge, internal state and so on. Whatever the environment we are considering, it can change, it is dynamic; changing environment greatly affects the agents' state and consequently their behavior in reaching goals.

We consider the cognitive system as the triad $<\mathrm{a}$, g, e $>$ : agent, goal, environment, and the environment is made up by other cognitive systems. Modeling a cognitive system implies using a recursive definition: a cognitive system is a system of systems, or better a society of systems.

What we are introducing is a new idea of intelligent agent, no more an indivisible computational unity but rather an entity made by a brain and a body. The portion of agent realizing the brain contains the capability of reasoning and discovering services offered by the environment and the goal to purse, the capability of storing experiences for future learning and consciousness.

In the past we analyzed and implemented goals and environment by using organizational structures and we provided means for engineering self-conscious robotic systems with a methodology highly grounded on a goal bases analysis of the system [2][6]. The main advantage of the proposed model is to have a design abstraction allowing to model and engineer all the entities involved in a biologically inspired system with a specific attention to the environment considered both as the internal and the external world of each entity pursuing a specific goal. We claim that this powerful design abstraction eases designing complex systems and aids in filling the gap between BICAs and implementation frameworks.

\section{References}

1. J. Anderson and C. Lebiere. The newell test for a theory of cognition. Behavioral and Brain Sciences, 26(5):587-601, 2003.

2. M. Cossentino, N. Gaud, V. Hilaire, S. Galland, and A. Koukam. Aspecs: an agentoriented software process for engineering complex systems. Autonomous Agents and Multi-Agent Systems, 20(2):260-304, 2010.

3. B. Goertzel, H. de Garis, C. Pennachin, N. Geisweiller, S. Araujo, J. Pitt, S. Chen, R. Lian, M. Jiang, Y. Yang, et al. Opencogbot: Achieving generally intelligent virtual agent control and humanoid robotics via cognitive synergy. In Proceedings of ICAI, volume 10, 2010.

4. J. Laird, A. Newell, and P. Rosenbloom. Soar: An architecture for general intelligence. Artificial intelligence, 33(1):1-64, 1987. 
5. A. Samsonovich. Toward a unified catalog of implemented cognitive architectures. In Proceedings of the 2010 conference on Biologically Inspired Cognitive Architectures 2010: Proceedings of the First Annual Meeting of the BICA Society, pages 195-244. IOS Press, 2010.

6. V. Seidita and M. Cossentino. From modeling to implementing the perception loop in self-conscious systems. International Journal of Machine Consciousness (IJMC), 2(2):289-306, 2010.

7. B. J. Shapiro S.C. The glair cognitive architecture. International Journal of Machine Consciousness, 2(2):307-332, 2010.

8. R. Sun. The clarion cognitive architecture: Extending cognitive modeling to social simulation. Cognition and multi-agent interaction, pages 79-99, 2006. 